Voix et Images

volxetimages

\title{
À la recherche du sens : De quoi t'ennuies-tu, Éveline?
}

\section{Ellen Reisman Babby}

Volume 14, numéro 3 (42), printemps 1989

Gabrielle Roy

URI : https://id.erudit.org/iderudit/200796ar

DOI : https://doi.org/10.7202/200796ar

Aller au sommaire du numéro

\section{Éditeur(s)}

Université du Québec à Montréal

\section{ISSN}

0318-9201 (imprimé)

1705-933X (numérique)

Découvrir la revue

\section{Citer cet article}

Reisman Babby, E. (1989). À la recherche du sens : De quoi t'ennuies-tu, Éveline?.

Voix et Images, 14(3), 423-432. https://doi.org/10.7202/200796ar d'utilisation que vous pouvez consulter en ligne.

https://apropos.erudit.org/fr/usagers/politique-dutilisation/ 
DOSSIER 423

\section{À la recherche du sens: De quoi t'ennuies-tu, Éveline?*}

\section{par Ellen Reisman Babby, Association for Canadian Studies in the United States, Washington, D.C.}

Le présent colloque a pour but de commémorer la vie et l'œuvre d'une grande écrivaine, Gabrielle Roy. Dans notre étude de l'héritage que nous lègue cette illustre auteure, il convient de nous arrêter à la dernière œuvre publiée avant sa mort, De quoi t'ennuies-tu, Éveline? Cette novella n'a reçu, jusqu'à présent, que peu d'attention de la part de la critique; pourtant ses personnages, son intrigue, son thème et sa structure narrative en font néanmoins un joyau de la production littéraire de Gabrielle Roy. L'œuvre, dédiée à son collègue et ami, François Ricard, a été publiée à la veille de sa mort et revêt de ce fait une importance toute particulière. Cette novella me servira de point de départ pour démontrer en quoi ce récit, court et dense à la fois, reflète les principaux aspects structuraux et thématiques de l'œuvre littéraire de Gabrielle Roy. Il sera fait mention de plusieurs cuvres de l'auteure dont Alexandre Chenevert, la Rivière sans repos, Un jardin au bout du monde et Ces enfants de ma vie.

De quoi t'ennuies-tu, Éveline? nous offre dès ses premières lignes les éléments caractéristiques de l'œuvre de Gabrielle Roy. Ces lignes nous révèlent le désir d'Éveline de partir, connâ̂tre autant que possible les merveilles de ce monde, traverser la vie en voyageur (p. 11), un désir pourtant refréné par son cœur enchaîné (p. 11). Ces phrases évoquent immédiatement plusieurs personnages familiers de l'auteure, entre autres Rose-Anna (Bonheur d'occasion), Christine (la Route d'Altamont et Rue Deschambault) et Alexandre Chenevert. En fait, la majorité des personnages de Gabrielle Roy ont cet ardent désir de voyage, de découverte et d'évasion d'un milieu contraignant.

Fait intéressant, le prologue de De quoi t'ennuies-tu, Éveline? est narré par la fille d'Éveline qui s'efface aussitôt son introduction terminée. La novella proprement dite est racontée par une tierce personne qui est omnisciente.

Après notre rencontre avec Éveline, nous apprenons qu'elle a reçu un télégramme énigmatique de son frère habitant la Californie. Le message est le suivant: Majorique à la veille du grand départ souhaite revoir Éveline. Argent suit. (p. 12) Le prologue se termine sur ce curieux message. C'est dans le décodage de ce message que tout le récit prend son envol. Les mots grand départ laissent Éveline perplexe. Son frère Majorique est-il sur le point de partir en

* Le texte de cette communication a été traduit de l'anglais par Jacinte Baillargeon. 
voyage ou, comme le lecteur le suppose, est-il sur le point d'affronter la mort? Après mûre réflexion, Éveline décide de se rendre en Californie pour connaître le sens de ce message. Dans notre voyage en autobus avec Éveline, nous reconnaissons plusieurs des personnages, des intrigues, des mises en scène, des structures et des thèmes propres à Gabrielle Roy. Ce texte, publié à la veille du grand départ (de Gabrielle Roy), constitue un épilogue tout indiqué à l'œuvre de l'auteure.

Que pouvaient signifier ces mots: à la veille du grand départ? Que Majorique était très malade, sur le point de mourir peut-être? Ou bien qu'il s'apprêtait à partir pour une autre destination tout simplement? (p. 13) La question du langage - sa signification - est déjà inscrite au tout début du texte.

La préoccupation du langage et de l'expression (comme je l'ai démontré dans une précédente analyse ${ }^{1}$ ) est un élément central des textes de Gabrielle Roy. En plusieurs occasions, le langage trahit le signifié. Les personnages s'en remettent souvent à des formes de communication non verbales. La gestuelle, la peinture, la sculpture et la musique sont au nombre de celles que chérissent les personnages régiens, surtout ceux qui essayent de communiquer dans un milieu peu familier. Martha, le personnage principal de Un jardin au bout du monde, nous en donne un bel exemple. Martha et Stepan Yaramko sont des immigrants ukrainiens établis dans un village éloigné du Nord canadien. Les nombreux efforts de Martha pour apprendre l'anglais la poussent même à étudier le catalogue du magasin Eaton. Mais elle doit abandonner, car le catalogue ne lui donne pas les outils linguistiques nécessaires pour traduire ses pensées intimes. Elle trouve finalement son expression dans in code non verbal, en l'occurrence un jardin, qu'elle cultive religieusement malgré l'hostilité du climat. Plus tard, Martha, atteinte d'un cancer, est confinée au lit et incapable de cultiver son jardin. C'est toutefois ce jardin, ce langage non verbal, qui nous raconte l'histoire de Martha qui «survit» grâce à lui. En fait, c'est la découverte du jardin qui donne le coup d'envoi à l'histoire de Martha.

Quant à Alexandre Chenevert et à Elsa (la Rivière sans repos), ils tentent désespérément de traduire leurs pensées, mais il leur manque les mots. Les scènes cruciales qui décrivent ces tentatives sont situées au centre de leurs récits respectifs.

Alexandre Chenevert est dépeint comme un commis de banque nerveux qui se débat à l'intérieur des limites du temps et de l'espace. Tout l'accable, les contraintes de son travail à la banque, les feux de signalisation et la fuite du temps. Cette prison métaphorique est envahissante. Des problèmes de santé poussent Alexandre à s'enfuir de la métropole trépidante qu'est Montréal. Il part à la campagne en autobus et se rend au lac Vert. Il y ressent un tel bien-être et une telle satisfaction qu'il éprouve le besoin d'exprimer ses pensées et décide d'écrire une lettre à un journal. Ses tentatives désespérées pour traduire ses pensées en langage sont relatées de main de maître:

1 Ellen Reisman Babby, The Play of Language and Spectacle: A Structural Reading of Selected Texts by Gabrielle Roy, Toronto, ECW Press, 1985. 
Que dire des livres qu'il avait apportés et dont l'auteur [...] continuait à charmer les longues soirées d'Alexandre. De l'encre, une plume, du papier, et lui aussi allait confier ses impressions!

[...]

Il dirait comment la paix vient, ensuite l' espoir... [...]

Tout cela n'était encore que dans le cœur d'Alexandre.

Sur la page blanche, il n'y avait rien.

[...]

Rien ne lui venait. [...]

Il se leva, arpenta la cabane [...] Il s'était remis à fumer: une soucoupe sur sa table était déjà remplie de mégots. [...]

[...] la beauté était en lui; nulle force au monde n'aurait pu lui arracher cette poignante certitude qu'il la possédait en son caur. Il ne lui manquait que les mots. Et comment se faisait-il qu'une émotion aussi profonde, aussi sincère, $n$ 'appelât point les moyens qui l'eussent rendue communicable?

[...]

[...] Alexandre se frappa le front: cette boîte rebelle devait au moins lui restituer ce qu'elle avait contenu. [...] Il frémit tout à coup d'impatience. [...]

[...] Les efforts de trois longues soirées lui avaient du moins appris la célérité avec laquelle il faut capter les pensées qui méritent d'être retenues.

Il trempa sa plume. Se penchant sur la feuille de papier, il tremblait de crainte autant que de détermination. Hélas, sous ses yeux remplis de stupeur, il vit sa propre pensée lui échapper, rentrer apparemment dans quelque trou dont c'était à peu près certain qu'il n'arriverait plus à la déloger. (AC, p. 250-253, nous soulignons)

Nous en venons à découvrir que la principale source du conflit qui angoisse Alexandre ne tient ni au temps ni à l'espace, mais plutôt au langage lui-même. Il est incapable de saisir ses pensées et de les transformer en mots. Son échec tragique face à l'expression écrite révèle une insatisfaction profonde à l'égard du langage comme moyen de communication. L'expression linguistique est donc décrite comme un système inadéquat de signification.

Dans une scène d'une similitude remarquable, Elsa, le personnage principal de la Rivière sans repos, veut utiliser le matériel moderne d'écriture que son oncle Ian a obtenu de Fort Chimo. Elle décide d'écrire à madame Beaulieu, l'épouse de l'agent de la paix en poste à Fort Chimo. Dans un effort désespéré pour traduire sa pensée en mots, Elsa prend conscience de la nature contraignante du système de signes linguistiques: 
Jamais elle n'aurait cru que cet effort usait autant. Rien qu'à force de tenir son crayon serré entre ses doigts elle en avait des crampes. L'esprit aussi était étrangement fatigué. Suivre ses pensées comme on suit la course des nuages, ou encore, indéfiniment, le fil de l'eau, était une chose: courir après, les traquer, les enfermer en des mots, en était une autre. Au beau milieu d' une phrase, elle s'arrêta, ne sachant plus comment s'en tirer [...] (RSR, p. 222-223, nous soulignons)

Dans De quoi t'ennuies-tu, Éveline?, le problème ne provient pas de la conversion d'une forme de langage en une autre, mais plutôt du code linguistique lui-même. En effet, la traversée du continent en autobus par Éveline devient une recherche du signifié. La protagoniste et ses compagnons de voyage proposent différentes interprétations du message linguistique ambigu. Éveline s'efforce de préciser le sens du message concernant Majorique en le transmettant à ses compagnons de voyage: Je me demande encore, dit-elle, ce que ces mots: le grand départ peuvent signifier. Qu' en pensez-vous? ajouta-t-elle en tendant le télégramme à sa voisine. (p. 20) Plusieurs passagers et le conducteur lui-même y vont de leur interprétation. Éveline se met ensuite à raconter des histoires à propos de son frère Majorique. Ses talents de conteuse se révèlent et plusieurs passagers tendent l'oreille pour l'écouter. Par son langage gestuel, elle parvient même à attirer l'attention des gens qui ne parlent pas français:

La physionomie, les gestes de cette petite vieille étaient si vivants que quelques-uns, qui ignoraient le français, furent peinés de ne pas comprendre ce qu' elle racontait et demandèrent à Mme Leduc de leur dire de quoi il s'agissait. Celle-ci traduisit donc une bonne partie de l' histoire, que les voisins transmirent d leur tour à ceux d'en avant et d'en arrière [...]. (p. 21-22)

Les initiés de l'œuvre de Gabrielle Roy reconnaîtront ici la structure narrative: c'est madame Leduc, la compagne de voyage d'Éveline, qui joue le rôle de narratrice et qui relate, à son tour, l'histoire de Majorique aux autres. Cette génération et re-génération du récit apparaissent dans plusieurs des textes de Gabrielle Roy. Les exemples les plus frappants se trouvent dans Rue Deschambault et la Rivière sans repos et méritent qu'on s'y arrête quelque peu.

Dans Rue Deschambault se trouve une histoire d'une grande intensité dramatique qui s'intitule «Le puits de Dunrea». L'histoire décrit l'arrivée d'immigrants ruthènes dans la région de Dunrea, au Canada. Narrée par Christine, l'histoire s'oriente autour de son père, Édouard, officier de colonisation qui aide les immigrants à s'installer. La première partie du récit fait état d'une colonie prospère; toutefois, au cours du récit, le destin des immigrants change du tout au tout lorsqu'un incendie dévaste leur colonie. La plupart s'en tirent indemnes mais, à leur retour à Dunrea, ils constatent qu'ils ont tout perdu. Édouard frôle la mort, ce qui donne au récit son intensité dramatique. Il se réfugie dans le puits de Dunrea dans un effort ultime pour se soustraire à l'incendie qui fait rage. Son combat est l'un des moments les plus bouleversants et les plus dramatiques de l'ouvrage de Gabrielle Roy. 
Au-delà des éléments thématiques du récit, nous découvrons, à l'étude de la structure narrative, que la version de l'histoire racontée par Christine est en réalité la re-génération d'un récit que son père Édouard avait fait à sa sœur Agnès. Christine relate donc une histoire qui a déjà été racontée à deux reprises, d'abord par Édouard, puis par Agnès. En y regardant de plus près, nous découvrons que la structure narrative de ce récit reflète le desserrement progressif des liens entre Christine et son père. La distance, malheureuse et irréductible, qui éloigne ces deux personnages est donc représentée à la fois par le thème et par la structure.

Un exemple de re-génération de récit dans une autre nouvelle de Gabrielle Roy, «Les satellites», mérite d'être souligné. Cette nouvelle, tirée de la Rivière sans repos, se situe à Iguvik, un lointain village inuit du Nord québécois. Au début de l'histoire, plusieurs habitants du village attendent l'arrivée d'un avion du gouvernement qui doit amener Deborah dans le Sud pour se faire soigner. Parmi eux se trouve Isaac, le patriarche du village. Il ne voit pas la nécessité de prolonger les souffrances de Deborah. 11 joue le rôle de conteur et relate la légende de la mort de La Vieille, une histoire bien connue de la plupart des personnes qui l'entourent. Le récit d'Isaac nous apprend que nous ne pouvons éviter la mort et qu'il nous faut l'affronter avec courage. Cette légende joue un rôle important dans l'histoire parce qu'il y est souvent fait allusion et qu'elle est répétée à plusieurs reprises. Nous apprenons que sa propre interprétation est elle-même issue d'une reprise ou d'une version «mythique» antérieure. Le mythe et la réalité se mêlent de façon inextricable malgré les efforts d'Isaac pour assurer la véracité du récit. Même si La Vieille et Deborah sont très différentes, elles se confondent à la fin du texte et, comme le titre l'évoque, deviennent des satellites, des mythes qui rôdent autour du village. Réalité et fiction et génération et re-génération du récit se mêlent en un art consommé. L'histoire se perpétue grâce à la narration ultérieure de l'histoire de Deborah.

Si nous nous reportons au texte de De quoi t'ennuies-tu, Éveline?, nous reconnaissons tout de suite l'importance des dernières paroles de Majorique: Vous serez autour de moi et vous vous raconterez des histoires... (p. 80) En fait, la deuxième tranche du récit se compose pour la majeure partie de la narration et de la re-narration d'histoires à propos de Majorique. Il y est fait référence au penchant de Majorique et d'Éveline pour les histoires et à leur talent de conteur. Dans un aparté maladroit quoique éclairé, le narrateur omniscient jette de la lumière sur la théorie de Gabrielle Roy à propos de la narration. Lorsque Éveline raconte une histoire aux enfants de Majorique, le narrateur esquisse les éléments clés d'une bonne histoire:

Répétée textuellement, son histoire n'aurait pas été assez vivante. Pour bien raconter, elle le savait, il fallait d'abord être prodigieusement captivé soi-même, et à cela on n'arrivait qu'a force de renouvellement [...] Elle ne pouvait ni ne voulait changer les faits, mais leur interprétation ne variait-elle pas à l'infini? Du reste, ce qui faisait une bonne histoire [...] c'était malgré tout la vérité: vérité des personnages, vérité des lieux, vérité des événements. (p. 75) 
Le conteur joue un rôle central dans l'œuvre tout entière de Gabrielle Roy. En effet, le besoin irrésistible de conter et de reconter des histoires peut y être considéré comme un thème caractéristique. Plusieurs personnages, dont Martha, Alexandre, Elsa et Éveline partagent ce besoin. Cette «urgence» de raconter nous porte à réfléchir sur l'extrait de Hamlet qui se trouve dans le texte régien le plus esthétique, la Montagne secrète. Pierre Cadorai est à bord d'un bateau qui l'emmène en Europe où il part étudier les ouvres des grands peintres européens. Il s'attarde sur un court extrait de Hamlet qu'il répète plusieurs fois. Ce passage précède immédiatement la mort de Hamlet:

If thou didst ever told me thy heart

Absent thee from felicity awhile,

And in this harsh world draw thy breath in pain

To tell my story.2

L'importance de cet extrait est claire. La piècequi jongle avec l'illusion et la réalité et qui remet en question le système de signes linguistiques s'impose comme sous-texte principal. L'urgent besoin qu'éprouve Hamlet de raconter son histoire, son obsession du langage et sa quête d'identité se retrouvent chez la majorité des personnages de Gabrielle Roy. Cet aspect de son œuvre n'a pas été pleinement étudié. Les commentateurs des œuvres de Gabrielle Roy ont quelque peu négligé le fait que beaucoup de ses personnages appartiennent à «l'âge de la parole». Les conteurs de Gabrielle Roy et surtout Isaac, Éveline et Elsa, reconnaissent que c'est par le fait de «nommer» qu'ils conserveront leur identité.

Tout au long de son périple en autobus, Éveline sera souvent perçue comme une «curiosité». Partie pour la Californie en plein hiver, elle porte un long manteau avec un col de fourrure, une grande écharpe, des gants de laine et des bottes fourrées. Le chauffeur de l'autobus et ses compagnons de voyage en parlent comme de la petite vieille dame habillée comme pour aller au pôle (p. 35). À mesure que l'autobus l'emmène vers le sud-ouest, les gens la remarquent à son étrange costume:

[...] elle se rendit compte qu' elle était remarquée de tous, et même que beaucoup de gens se mettaient à sourire en la voyant. Alors, parmi tous ces gens en toilette claire, elle se vit, elle, dans son gros manteau d'hiver, ses bottes fourrées aux pieds, et partit à rire gaiement. (p. 55)

Tandis que le narrateur adopte ici le point de vue des compagnons de voyage d'Éveline, la perspective est généralement celle d'Éveline qui se fraie un chemin dans un monde peu familier. Son émerveillement à la vue de la Sierra Nevada est délicatement présenté. Ses premières impressions de Las Vegas méritent d'être citées:

2 Hamlet, acte V, scène II. Reproduit par Gabrielle Roy, dans la Montagne secrète, p. 147. 
Il y eut un bref arrêt dà Las Vegas. Des milliers de lumières vives, des enseignes multicolores qui s'allumaient et s'éteignaient sans cesse, dessinant dans le ciel des images absurdes, d'énormes chapeaux de cow-boys, des chiens à la course [...] Éveline clignait des yeux, se croyant le jouet de quelque monstrueuse folie. Après les suppliciés du désert, que faisaient ici ces lumières dansantes? (p. 56)

Le récit de la découverte de l'océan par Éveline est tout aussi remarquable. Olaf, le vieil ami de Majorique, nous fait part du regret qu'éprouvait ce dernier de savoir que sa sœur n'avait jamais vu l'océan. Lorsque Éveline se retrouve en Californie et rencontre les membres de la famille de Majorique, son neveu promet de l'emmener voir l'océan. Mais il faut d'abord enterrer Majorique. Après avoir gravi la colline jusqu'à la chapelle et s'être rendue au lieu choisi par Majorique pour son demier repos, Éveline jette un regard autour d'elle au moment où l'on enterre son frère:

Alors, levant la tête, Éveline aperçut en bas, très loin, miroitant sous le soleil, une surface calme, brillante et infinie. Qu'était-ce? Un mirage? Une illusion? [...] De nouveau, elle regarda briller ce lointain uni, immense, sans rides, plus exaltant dans son mystère que tout ce qui l'avait saisie d'émotion pendant sa vie entière. Et cependant, ce n'était rien; non, rien que de l' uni, de l'infini, le calme parfait [...] Alors, ayant suivi son regard et lisant sur son visage l'expression du doute et de l'espérance, le petit Edwin lui pinça le bras et chuchota - Oui, Auntie dear, c'est l'océan. (p. 94-95)

L'histoire se termine sur cette émouvante découverte de l'océan par Éveline.

Cette découverte et les impressions qu'inspire à Éveline la ville trépidante de Las Vegas nous rappellent le jeu des impressions de plusieurs autres écrits de Gabrielle Roy et en particulier des nouvelles qui précèdent le roman la Rivière sans repos. Ainsi, dans «Les satellites», nous partageons avec Deborah, une habitante de l'aride toundra, la touchante découverte d'un arbre dont la vue l'accable:

Elle s'approcha d'un des érables un peu comme on s'approche avec précaution d' un être vivant, à ne pas effaroucher. Elle tendit d'abord la main pour le toucher délicatement, du bout des doigts. C'était comme si elle cherchait à l'apprivoiser. Ensuite elle leva vers lui un regard réjoui tout en l'écoutant bruire. Elle finit par passer un bras autour du tronc et, y appuyant sa joue, elle demeura longtemps immobile à contempler haut dans le ciel la grande masse des feuilles que le vent agitait doucement. (RSR, p. 37)

L'auteure utilise la même technique pour montrer la fascination qu'exercent sur Deborah le savon et la douche chaude ainsi que les premières impressions de Barnaby à propos du téléphone dans la nouvelle «Le téléphone». Une histoire teintée d'humour qui souligne l'aliénation de Barnaby face à la culture des Blancs. 
Gabrielle Roy utilise ici le processus de défamiliarisation ou de transformation du familier en étrange ${ }^{3}$. Elle décrit les objets, les villes comme s'ils étaient vus pour la première fois. L'accent est mis sur l'acte de la perception: le vu l'emporte sur le connu. Le narrateur adopte parfois le point de vue du personnage innocent tandis qu'à d'autres moments, il adopte la perspective de la société «moderne». L'auteure nous convie à savourer ces deux perspectives, la nôtre qui est ancrée dans la société «moderne» et celle d'un personnage naîf.

Après avoir eu l'occasion de rencontrer les membres de la famille de Majorique, Éveline veut en savoir plus long sur la vie qu'a menće son frère en Californie. Par ailleurs, les enfants et petits-enfants de Majorique veulent connaître ses origines. Tout comme Isaac dans «Les satellites», Éveline redevient la conteuse et assure le lien essentiel entre le passé et le présent. Friande de l'humour de son défunt frère, Éveline regarde son décès et en particulier son cortège funèbre du point de vue de ce dernier.

Éveline pensa que Majorique, en tête du défilé, devait sourire de triomphe. $N$ 'avait-il pas réalisé aujourd' hui le plus beau tour de sa vie? [...] La montagne verdoyante, les fleurs exquises, ce ciel d'été quand ce devrait être l' hiver, c'en était trop sans doute pour Éveline. Elle pensa un moment: «Majorique n'est pas mort. Il s'amuse à nous réunir de tous les coins du monde pour cette promenade magnifique.» (p. 93, nous soulignons)

Rappel de ses origines: [...] ce ciel d'été quand ce devrait être l'hiver. Éveline pense à l'hiver de sa terre natale au moment où elle enterre son frère. Semblable en cela au mouvement actuel de la littérature québécoise où les personnages sortent de l'espace littéraire du Québec et élargissent leurs horizons, il s'agit de l'une des rares nouvelles de Gabrielle Roy où le récit se déroule à l'extérieur du Canada. Par contraste avec Rose-Anna et sa mémorable visite au lieu de ses origines (la maison de campagne de sa mère, madame Laplante, au Québec), Éveline doit traverser une frontière et franchir une grande distance pour découvrir la patrie d'adoption de son frère bien-aimé. Alors que tant d'histoires de Gabrielle Roy portent sur la situation de l'immigrant au Canada et, dans plusieurs cas, du Québécois ailleurs au Canada, l'auteure nous expose ici la situation d'un Canadien français qui a émigré. L'évolution mérite d'être signalée.

La recherche des origines est un élément central qui revient fréquemment dans l'œuvre de Gabrielle Roy. Qu'on se rappelle Elsa, dans la Rivière sans repos, qui entreprend un voyage mémorable au Vieux Fort Chimo et regarde les épitaphes des pierres tombales. Elle essaie désespérément de retracer son patrimoine: Elle s'arrêtait ça et là pour déchiffrer difficilement ce qui en restait. Elle saisit un nom tout juste encore déchiffrable. (RSR, p. 202) Malheureusement, les épitaphes sont pratiquement effacées et la quête d'identité d'Elsa est un échec.

3 Pour une discussion plus élaborée de cet aspect, voir. E. Reisman Babby, The Play of Language and Spectacle, p. 29; pour une discussion du processus de défamiliarisation, voir Russian Formalist Criticism, Reagan, University of Nebraska Press, 1967, p. 12. 
Le retour aux origines de Rose-Anna, dont nous avons déjà parlé, joue un rôle important dans Bonheur d'occasion. Le roman Alexandre Chenevert présente lui aussi une scène analogue. La partie centrale de l'ouvrage, soit lorsque Alexandre quitte Montréal pour le lac Vert, marque un retour à la nature. Le lac Vert apparaît comme un lieu idyllique éloigné du tumulte de la métropole.

La recherche des origines pénètre également le niveau des personnages dans les textes de Gabrielle Roy. Partout, dans ses romans comme dans ses nouvelles, des personnages ont perdu leurs parents naturels: Jean Lévesque est orphelin, Alexandre fait sans cesse allusion à la mort de sa mère, Elsa n'a pas de père et Jimmy (la Rivière sans repos) ne connaîtra jamais l'identité de son père. Qui plus est, l'absence de l'image du père hante nombre d'histoires de Rue Deschambault et de la Route d'Altamont. Fait significatif, la dernière œuvre publiée du vivant de Gabrielle Roy, De quoi t'ennuies-tu, Éveline?, se termine par la mort de la figure paternelle, Majorique.

Dans De quoi t'ennuies-tu, Éveline?, la préoccupation à l'égard des origines est transformée: Majorique, comme nous l'avons déjà dit, est enterré loin de sa terre natale. Nous pensons à d'autres personnages d'immigrants créés par Gabrielle Roy, comme Sam Lee Wong, Martha, Stepan, les Demetrioff et d'autres, dont les origines, tout comme celles de Majorique, resteront inconnues de leur entourage. Comme le signifiant linguistique qui trahit souvent les personnages, le problème des origines est profondément enraciné et touche à tous les niveaux de narration des auvres de Gabrielle Roy.

Le texte De quoi t'ennuies-tu, Éveline? apparaît de ce fait riche et empreint d'émotion. Sur les plans de la structure, de la thématique et de la narration, cette novella renferme tous les éléments clés de l'œuvre littéraire de Gabrielle Roy. En fait, vu sous l'angle de la situation et du traitement de l'espace, on peut dire que ce texte se rapproche plus de la période contemporaine de la littérature québécoise caractérisée par ce que l'on a appelé la nord-américanisation de la littérature du Québec:

This is not a process of Americanization, for Québec literature, by its language and its preoccupation, is still very different from the literature of the United States. But the fascination which France exerted on generations of Québec authors is being replaced in some quarters with a new attentiveness to the North American past of Québec and a new reading of American authors. 4

L'attrait universel et la complexité sous-jacente rendent les œuvres de Gabrielle Roy dignes d'une étude approfondie. Lorsque nous parcourons en une

4 Jonathan Weiss, French-Canadian Literature, à paraître au printemps 1989 dans les ACSUS Paper Series. Traduction: Ce n'est pas un processus d'américanisation que vit la littérature québécoise, car elle est, par sa langue et ses préoccupations, encore très différente de la littérature américaine. Mais la fascination exercée par la France sur des générations d'auteurs québécois a été remplacée dans certains domaines par un nouvel intérêt pour le passé nord-américain du Québec et par une nouvelle lecture des auteurs américains. 
promenade magnifique l'œuvre littéraire de Gabrielle Roy et la regardons à la lumière des tendances actuelles de la littérature canadienne-française, nous pouvons évaluer pleinement l'art consommé de cette écrivaine de renommée mondiale et reconnaître la perte énorme qui est la nôtre depuis son décès.

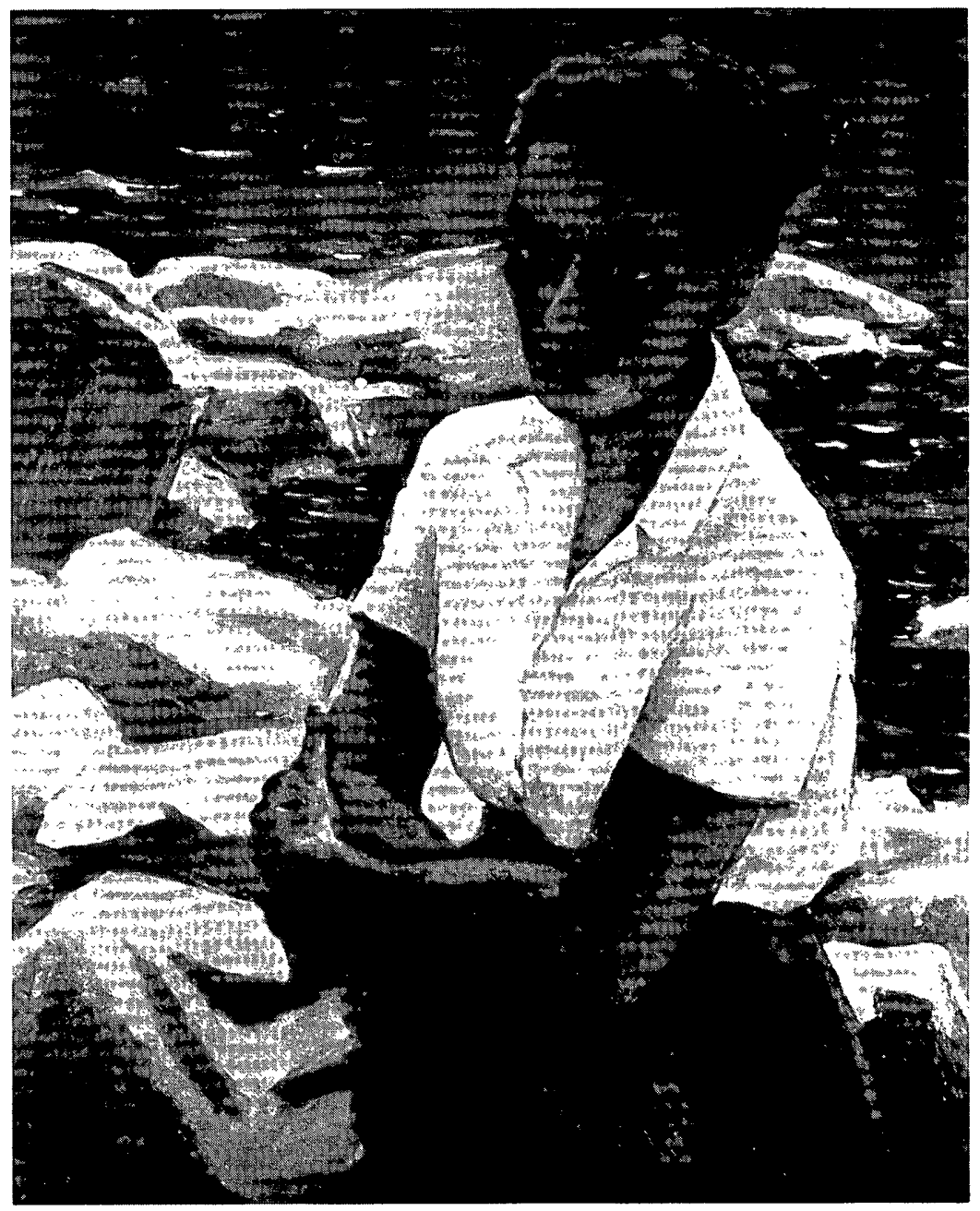

